\title{
Mycobacterial Dormancy Regulon Protein Rv2623 as a Novel Biomarker for the Diagnosis of Latent and Active Tuberculous Meningitis
}

\author{
Ruchika K. Jain, ${ }^{1}$ Amit R. Nayak, ${ }^{1}$ Aliabbas A. Husain, ${ }^{1}$ \\ Milind S. Panchbhai, ${ }^{1}$ Nitin Chandak, ${ }^{1}$ Hemant J. Purohit, ${ }^{2}$ Girdhar M. Taori, ${ }^{1}$ \\ Hatim F. Daginawala, ${ }^{1}$ and Rajpal S. Kashyap ${ }^{1}$ \\ ${ }^{1}$ Biochemistry Research Laboratory, Central India Institute of Medical Sciences, Bajaj Nagar, Nagpur 440010, India \\ ${ }^{2}$ Environmental Genomics Unit, National Environmental Engineering Research Institute, Nehru Marg, Nagpur 440020, India
}

Correspondence should be addressed to Rajpal S. Kashyap; raj_ciims@rediffmail.com

Received 10 June 2013; Revised 28 August 2013; Accepted 29 August 2013

Academic Editor: Silvia Persichilli

Copyright (C) 2013 Ruchika K. Jain et al. This is an open access article distributed under the Creative Commons Attribution License, which permits unrestricted use, distribution, and reproduction in any medium, provided the original work is properly cited.

\begin{abstract}
The present study was designed to investigate Rv2623 antigen, a major dormancy regulon protein of Mycobacterium tuberculosis (MTB) in CSF of suspected latent and active tuberculous meningitis (TBM) patients. A total of 100 CSF samples from TBM $(n=31)$, suspected latent TBM $(n=22)$, and suitable noninfectious control subjects $(n=47)$ were collected and evaluated for Rv2623 antigen level using ELISA protocol. A significantly high $(P<0.05)$ mean absorbance was observed in samples of suspected latent TBM and active TBM patients as compared to non-TBM control patients. However, no significant difference in Rv2623 level was observed between suspected latent TBM and TBM patients. Our preliminary findings suggest that Rv2623 may be useful as a potential biomarker for the diagnosis of the latent as well as active TBM infection. Futher evaluation of this biomarker in large number of samples is therefore needed to confirm the result.
\end{abstract}

\section{Introduction}

Tuberculosis $(\mathrm{TB})$ remains a major health problem worldwide [1]. Tuberculous meningitis (TBM) is among the most fatal forms of extrapulmonary tuberculosis (EPTB) accounting for $70-80 \%$ of all neurological TB cases [2-4]. The development of TBM infection occurs with infection of meninges with tuberculous bacilli, resulting in formation of tuberculous lesions (Rich foci). Although tuberculous bacilli can remain dormant in subependymal surface of the brain for years [5-7], however, reactivation may occur due to rupture or growth of small lesions in the meninges producing active infection $[8,9]$. Latent tuberculous meningitis (LTBM) continues to pose a diagnostic problem, as it remains nonsymptomatic and shows only inflammatory changes in cerebrospinal fluid (CSF) [10]. Biomarkers aiding in early diagnosis of LTBM are therefore urgently needed for early initiation of treatment and also to avoid further neurological complication and reactivation to active TBM infection.

Rv2623 is a major dormancy regulon protein of Mycobacterium tuberculosis (MTB) which is involved in promotion of mycobacterial transition to latency [11]. Although many studies suggests role of Rv2623 as a major diagnostic marker for latent infection, there are no studies reported involving evaluation of Rv2623 in CSF of suspected latent and active TBM patients. Keeping the existing situation in mind, the present study was therefor designed with an objective to investigate diagnostic utility of Rv2623 in CSF samples of suspected latent and TBM patients for the diagnosis. Apart from that, to further investigate the utility of Rv2623 as a prognostic maker in suspected LTBM cases, telephonic followup was taken for a period of two years to monitor conversion into active TBM cases. 


\section{Materials and Methods}

2.1. Study Subjects. A total of 100 CSF samples were collected from patients of infectious neurological disorders admitted to the Neurology Department of Central India Institute of Medical Sciences (CIIMS), Nagpur. The average age of the patients ranged between 10 and 50 years, and there were 54 males and 46 females. Patients were classified into three different groups based on their clinical profile.

2.2. TBM Group $(n=31)$. Diagnosis of TBM was based on the clinical features, which included subacute or chronic fever and signs of meningeal irritation with or without other features of central nervous system (CNS) abnormality. All patients recruited were positive for acid-fast bacilli and/or MTB CSF culture and started on antituberculous drugs treatment (ATT). CSF findings in these patients included increased protein levels, decreased glucose (CSF/blood glucose ratio, $<0.5$ ), and pleocytosis with lymphocyte predominance. All patients were culture negative for other microorganisms.

2.3. Suspected Latent TBM Group $(n=22)$. This category included patients having exposure with active TB subjects. Patients having clinical features such as subacute or chronic fever and signs of meningeal irritation with or without other features of CNS abnormality were included in this category. CSF findings in these patients included increased protein levels and decreased glucose (CSF/blood glucose ratio, $\leq 0.5)$. CSF cultures were negative for MTB and other microorganisms. AFB was also negative in these patients.

2.4. Non-TBM Noninfectious Control Group $(n=47)$. This category included patients with no clinical symptoms suggestive of meningitis, normal CSF protein and sugar level with no evidence of CNS or extra-CNS tuberculosis. Patients included in this category had no exposure to TB cases and were negative for MTB CSF culture and acid-fast bacilli.

Periodically, telephonic followup was taken from suspected LTBM cases for a period of two years based on initial Rv2623 positivity in their CSF samples. Out of 22 suspected cases, followup of was taken from 16 suspected LTBM cases to know persistence of any existing complications during the two-year period. Followup was not available in 6 cases due to unavailability of their records and due to other ethical reasons.

2.5. Specimen. CSF samples were collected by standard lumbar puncture. Routine CSF examination, Gram's staining, India ink staining, and acid-fast bacilli staining were done in every CSF sample. All the samples were stored at $-20^{\circ} \mathrm{C}$ until further analysis.

2.6. Detection of Rv2623 Antigen in the CSF Sample. Detection of Rv2623 antigen in the CSF samples was done by indirect ELISA protocol as described by Kashyap et al. and Mudaliar et al. [3, 4]. Briefly, $100 \mu \mathrm{L}$ of CSF sample (1:5 diluted in phosphate buffered saline (PBS) was coated on the wells of microtiter plates and incubated for $90 \mathrm{~min}$ at $37^{\circ} \mathrm{C}$. The wells were then washed once with PBST (PBS tween-20) and blocked with blocking buffer (0.5\% BSA in $\mathrm{PBST}$ ) and incubated at $37^{\circ} \mathrm{C}$ for $60 \mathrm{~min}$. After blocking, wells were washed three times with PBST followed by addition of $100 \mu \mathrm{L}$ monoclonal antibody (1:2000 dilutions in PBST) against Rv2623 antigen (Biotech, India). After $45 \mathrm{~min}$ of incubation, the wells were washed three time with the PBST and $100 \mu \mathrm{L}$ secondary antibody, and affinity purified anti-mouse IgG (1:5000 dilution in PBST) conjugated to horseradish peroxides (Genei, Bangalore, India) was added to wells and incubated at $37^{\circ} \mathrm{C}$ for $45 \mathrm{~min}$. After incubation, the wells were washed four times extensively with PBST followed by addition of $100 \mu \mathrm{L}$ of $\mathrm{TMB} / \mathrm{H}_{2} \mathrm{O}_{2}$ substrate and incubated at $37^{\circ} \mathrm{C}$ for $10 \mathrm{~min}$. The reaction was stopped with addition of $100 \mu \mathrm{L}$ of $2.5 \mathrm{~N} \mathrm{H}_{2} \mathrm{SO}_{4}$, and the absorbance of colour in each well was read at $450 \mathrm{~nm}$. Each sample was tested in triplicate.

2.7. Statistical Analysis. All the statistical analysis was performed using MedCalc statistical software (version 10.0.1). Comparison of baseline characteristics of TBM and suspected LTBM patients was done using chi-square test for the comparison of two proportions. Similarly, CSF characteristic of the TBM, suspected LTBM, and non-TBM control individuals was done using student's $t$-test, respectively. Mean absorbance of MTB specific Rv2623 antigen in CSF samples of TBM and LTBM and non-TBM control group was compared using Mann-Whitney $U$ test. Cut-off value for the Rv2623 antigen detection assay and its sensitivity and specificity were calculated by receiver operating characteristics (ROC) curve analysis. Graph of respective data was prepared using Prism (version 5) software (GraphPad Software, Inc. San Diego, CA). A $P$ value of $<0.05$ was considered statistically significant for all the analyses.

\section{Results}

The baseline characteristics of active and suspected latent TBM patients are shown in Table 1. No significant difference in baseline characteristics was found between active TBM and suspected LTBM patients with exception of headache and vomiting $(P<0.05)$.

Table 2 depicts the CSF characteristics in the three study groups. Increased levels of total cell count and protein were observed in samples of TBM and suspected LTBM patients as compared to non-TBM control group. However, significant increase $(P<0.05)$ in the above characteristics was observed only in TBM patients. Similarly, there was significant decrease $(P<0.05)$ in the levels of CSF sugar and sugar/parallel blood sugar ratio among TBM and suspected LTBM patients as compared to non-TBM control group. However, decrease was significantly more $(P<0.05)$ in TBM patients as compared to suspected LTBM and control group.

Figure 1 shows scatter plot of absorbance value of Rv2623 protein in CSF samples of TBM, suspected LTBM, and non-TBM control individuals. Comparison of the mean absorbance indicated significantly high Rv2623 levels $(P<$ $0.05)$ in TBM $(0.64 \pm 0.09)$ and suspected LTBM $(0.65 \pm 0.14)$ 
TABLE 1: Baseline characteristics of active TBM $(n=31)$ and suspected LTBM cases $(n=22)$.

\begin{tabular}{|c|c|c|}
\hline Characteristics & $\begin{array}{c}\text { Active TBM } \\
N(\%)\end{array}$ & $\begin{array}{c}\text { Suspected LTBM } \\
N(\%)\end{array}$ \\
\hline \multicolumn{3}{|l|}{ Age } \\
\hline$<18$ & $4(13)$ & $5(23)$ \\
\hline $18-40$ & $21(68)$ & $11(50)$ \\
\hline$>40$ & $6(19)$ & $6(27)$ \\
\hline \multicolumn{3}{|l|}{ Gender } \\
\hline Male & $10(32)$ & $10(45)$ \\
\hline Female & $21(68)$ & $12(54)$ \\
\hline \multicolumn{3}{|l|}{ Headache } \\
\hline Yes & $21(68)^{*}$ & $8(36)$ \\
\hline \multicolumn{3}{|l|}{ Fever } \\
\hline Yes & $22(71)$ & $10(45)$ \\
\hline \multicolumn{3}{|l|}{ Neck stiffness } \\
\hline Yes & $4(13)$ & 0 \\
\hline \multicolumn{3}{|l|}{ Vomiting } \\
\hline Yes & $19(61)^{*}$ & $6(27)$ \\
\hline \multicolumn{3}{|c|}{ Abnormal behavior } \\
\hline Yes & $3(10)$ & $3(14)$ \\
\hline \multicolumn{3}{|l|}{ Unconsciousness } \\
\hline Yes & $6(19)$ & $9(41)$ \\
\hline \multicolumn{3}{|l|}{ Drowsiness } \\
\hline Yes & $5(16)$ & $3(14)$ \\
\hline \multicolumn{3}{|l|}{ Seizures } \\
\hline Yes & $6(19)$ & $6(27)$ \\
\hline \multicolumn{3}{|l|}{ Nausea } \\
\hline Yes & $3(10)$ & $1(4)$ \\
\hline \multicolumn{3}{|l|}{ Blurred vision } \\
\hline Yes & $4(13)$ & $1(4)$ \\
\hline \multicolumn{3}{|c|}{ Weakness in limbs } \\
\hline Yes & $5(16)$ & $8(36)$ \\
\hline
\end{tabular}

TABLE 2: The CSF characteristics of the non-TBM control, suspected LTBM, and TBM patients, respectively.

\begin{tabular}{lccc}
\hline $\begin{array}{l}\text { CSF } \\
\text { characteristics }\end{array}$ & $\begin{array}{c}\text { Non-TBM } \\
\text { control }\end{array}$ & $\begin{array}{c}\text { Suspected } \\
\text { LTBM }\end{array}$ & TBM \\
\hline Total cell count & $69 \pm 165$ & $92 \pm 135$ & $131 \pm 152^{*}$ \\
Sugar & $66.01 \pm 31.17$ & $50.98 \pm 13.41^{*}$ & $36.23 \pm 24.22^{* @}$ \\
$\begin{array}{l}\text { Protein } \\
\text { Parallel blood }\end{array}$ & $78.33 \pm 50.50$ & $88.16 \pm 91.45$ & $153.47 \pm 201.68^{*}$ \\
sugar & $123.35 \pm 38.62$ & $113.91 \pm 28.57$ & $110.29 \pm 21.98$ \\
$\begin{array}{l}\text { Sugar/parallel } \\
\text { blood sugar ratio }\end{array}$ & $0.55 \pm 0.2$ & $0.52 \pm 0.17$ & $0.34 \pm 0.25^{* @}$ \\
\hline
\end{tabular}

Note: ${ }^{*} P>0.05$ versus control, ${ }^{@} P>0.05$ versus LTBM.

patients as compared to non-TBM control $(0.37 \pm 0.07)$ individuals. No significant difference was observed in mean absorbance between TBM and suspected LTBM subjects.

Table 3 shows diagnostic sensitivity, specificity, positive predictive value (PPV), and negative predictive value (NPV)

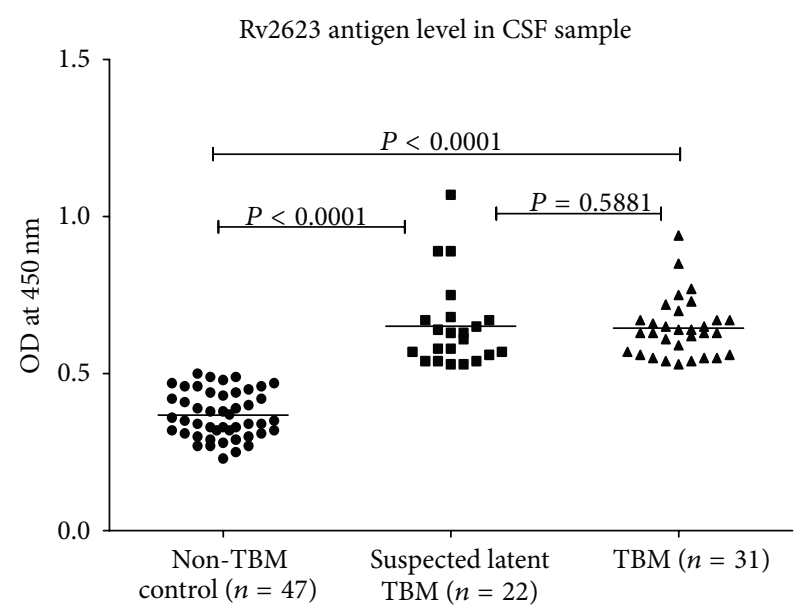

FIGURE 1: Shows scatter plot of the absorbance value of Rv2623 in the CSF samples of non-TBM control, suspected LTBM, and TBM patients, respectively.

of Rv2623 estimation for the diagnosis of TBM and suspected LTBM patients in the CSF samples. Diagnostic sensitivity of Rv2623 estimation in TBM and suspected LTBM patients was found to be $90.32 \%$ and $77.27 \%$, respectively, with a specificity of around $100 \%$ for both. PPV of the test was $100 \%$ in both TBM and suspected LTBM patients, while the NPV was higher in TBM patients (94\%) then the suspected LTBM patients (90\%). Among the three cases false negative for Rv2623 antigen in active TBM group, one was an old case of TBM along with encephalopathy, while the other two cases were old cases of multiple granuloma of brain tuberculosis. Clinical diagnosis in five, false negative cases for Rv2623 in suspected LTBM group revealed that two cases were of that of encephalitis with viral meningitis, while the other three were cases of calcified granuloma and CNS demyelination, respectively.

Figure 2 shows a flow chart analysis of follow-up samples and their conversion with respect to Rv2623 levels in CSF. Out of 22 suspected cases, followup was available in 16 cases. Four cases out of 16 (25\%) showed conversion to active TBM. Two cases were among those who responded well on starting ATT, while the remaining two cases expired during followup.

\section{Discussion}

Despite the recent advances in TB diagnostic tools, diagnosis of TBM still poses a major problem $[12,13]$. The signs and symptoms, along with routine CSF and radiographic finding in patients with CNS tuberculosis, are often inadequate for making confirmative diagnosis [13]. Identification of a new biomarker for rapid, confirmatory, and differential diagnosis of TBM is therefore urgently needed.

In the present study, we evaluated diagnostic potential of Rv2623, a mycobacterial dormancy regulon protein [14] in CSF samples of active TBM, suspected LTBM, and control individuals. Our results revealed significantly high Rv2623 level in TBM and suspected LTBM patients as compared to non-TBM control individuals. However, no significant 
TABLE 3: Sensitivity, specificity, positive predictive value (PPV), and negative predictive value (NPV) for Rv2623 Ag ELISA assay in TBM and suspected LTBM cases.

\begin{tabular}{lcccccc}
\hline Group & TBM $(n=31)$ & $\begin{array}{c}\text { Control } \\
(n=47)\end{array}$ & $\begin{array}{c}\text { Sensitivity } \\
\%(95 \% \mathrm{CI})\end{array}$ & $\begin{array}{c}\text { Specificity } \\
\%(95 \% \mathrm{CI})\end{array}$ & $\begin{array}{c}\text { PPV } \\
\%(95 \% \mathrm{CI})\end{array}$ & $\begin{array}{c}\text { NPV } \\
\%(95 \% \mathrm{CI})\end{array}$ \\
\hline $\begin{array}{l}\text { Rv2623 positive } \\
\text { Rv2623 negative }\end{array}$ & 28 & 0 & 90.32 & 100 & 100 & 94 \\
\hline & 3 & 47 & $(74.22-97.85)$ & $(92.38-100)$ & $(87.54-100)$ & $(83.43-98.68)$ \\
\hline Rv2623 positive & Suspected LTBM $(n=22)$ & & & & & 100 \\
Rv2623 negative & 17 & 0 & 77.27 & 100 & $(80.33-100)$ & $(78.96-96.77)$ \\
\hline
\end{tabular}

Note: sensitivity, specificity, PPV, and NPV were calculated on the basis of cut-off value ( $\geq 0.54)$ obtained from ROC curve analysis.

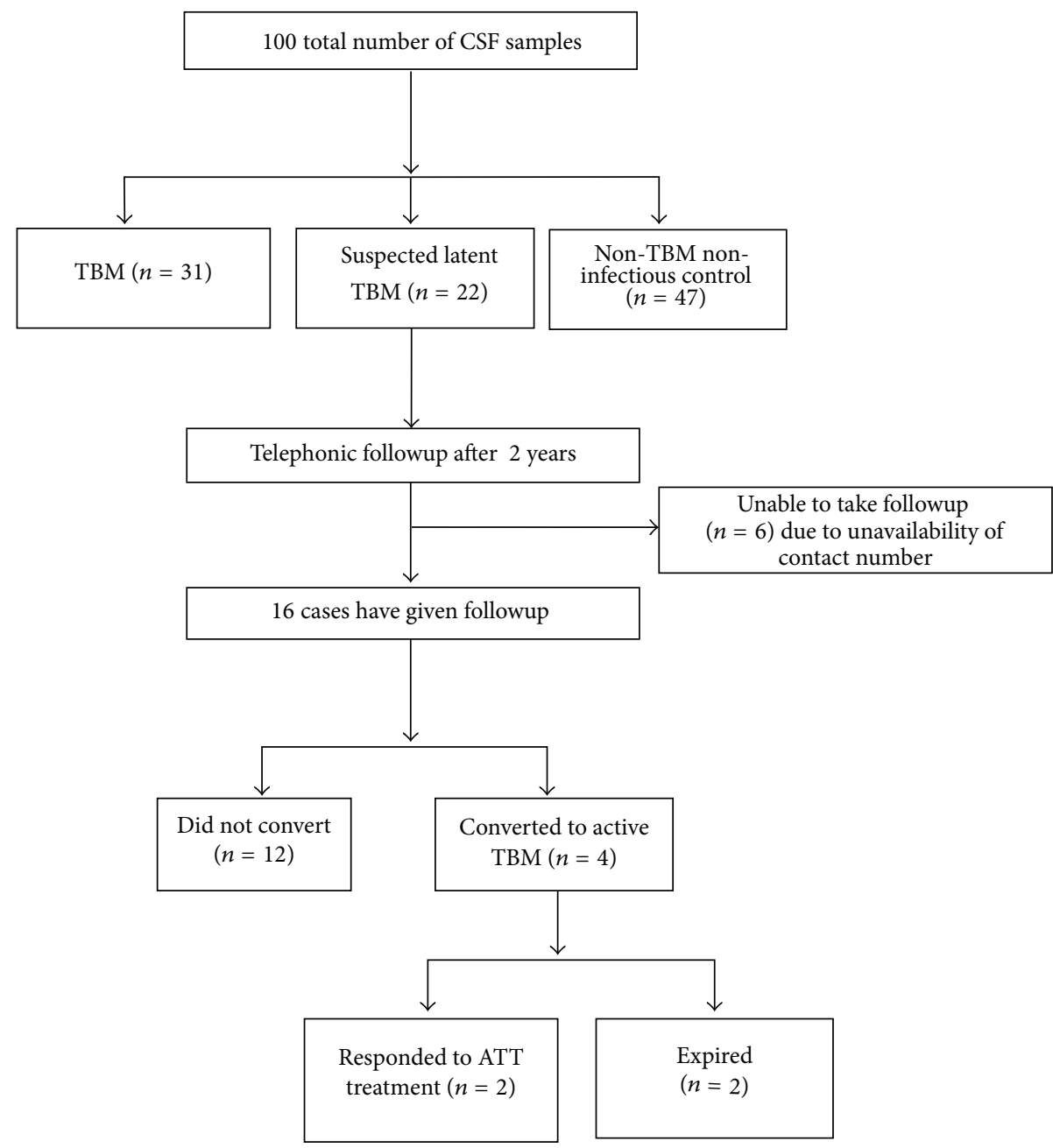

FIGURE 2: Flow chart analysis of two-year followup of Suspected LTBM cases and their conversion into active TBM.

difference in Rv2623 level was observed between TBM and suspected LTBM subjects. Overall sensitivity of Rv2623 estimation in TBM and suspected LTBM patients was $90.32 \%$ and $77.27 \%$, respectively, with $100 \%$ specificity.

Rv2623 is a member of the universal stress proteins family and is reported to protect mycobacteria from multiple stress conditions like hypoxia, nitrosative stress, low $\mathrm{pH}$ levels, and so forth [15-17]. Schuck et al. in their study evaluated twenty-nine $M$. tuberculosis dormancy associated antigens including Rv2623 and found that RV2623 induced significantly stronger T-cell responses in LTBI as compared to TB patients [18]. Similarly, it is found that a level of Rv2623 protein significantly increases following phagocytosis by macrophages in lungs of chronically infected mice. In another study, Monahan et al. showed increased expressions of six abundant proteins (including Rv2623) inside MTB 
infected macrophages, supporting the fact that Rv2623 may promote transition of mycobacteria to latency [19]. Interestingly, Rv2623 knockout mutant of virulent M. tuberculosis failed to establish a chronic persistent infection, suggesting the role of Rv2623 in regulation and establishment of a persistent infection [11]. Florczyk et al. reported that Rv2623 was strongly expressed in bacteria from resting cultures and absent in shaking cultures, supporting the role of Rv2623 in the anaerobic survival of $M$. tuberculosis inside the granuloma in the dormant state [20]. In our earlier studies, we had also evaluated the role of Rv2623 for diagnosis of latent TB infection (LTBI) (unpublished data), and our finding are in agreement with the earlier published reports. As mentioned elsewhere, there are various reports which suggest Rv2623 as a latent specific biomarker in pulmonary TB; however, to the best of our knowledge, this is the first study to report evaluation of Rv2623 antigen in the CSF samples for the diagnosis of latent and active TBM infections. Result from the current study supports our earlier findings that Rv2623 level increases after MTB infection. In the current study, patients included under the suspected LTBM group had neurological complications and were culture negative for any other organism. As mentioned, despite absence of any clinical symptoms of TBM and culture negativity in the body fluid, we observed increased Rv2623 levels in 77.27\% (17/22) of cases, indicating the importance of Rv2623 evaluation in CSF for diagnosis LTBM infection. The main function of Rv2623 is to regulate the bacillary growth inside the tuberculous lesions (Rich foci) located in any part of brain where organism may remain dormant for a long period of time [5-9]. Thus, evaluation of Rv2623 in CSF samples may be very useful for the diagnosis of persistent LTBM infection in such patients. Further role of Rv2623 antigen in LTBM infection can be evaluated by doing long-term followup of these subjects. Similarly, it will be interesting to see the conversion of LTBM cases defined in this test to active TBM cases.We also evaluated case-wise association of Rv2623 with different CSF findings. We observed that absorbance of Rv2623 positively correlated with total cell count and protein; similarly, a negative correlation with sugar and sugar/parallel blood sugar ratio was observed both in TBM and LTBM groups (Supplementary Figures 1-2 available online at http://dx.doi.org/10.1155/2013/309816). We did not find any correlation between Rv2623 absorbance and CSF finding in control group (Supplementary Figure 3). This confirms our findings that Rv2623 may be a useful diagnostic biomarker for the diagnosis of LTBM and TBM infections.

Similarly, we have also evaluated the prognostic utility of Rv2623 by taking telephonic followup of 22 suspected TBM cases based on earlier Rv2623 positivity. After two years, followup of 16 cases was available. About 25\% (4/16) showed conversion, of which two cases were among those that expired, while the other two cases were started on ATT and responded well to treatment. Although the percentage of conversion reported was much less, it still suggests important findings, as we got only 16 cases for followup, which is altogether a very low number. Still, four cases showing conversion were among those who earlier had no signs and symptoms of TBM, but showed higher Rv2623 levels in their CSF. These results suggest the utility of Rv2623 as an important marker for diagnosis and prognosis in latent and active TBM cases.

Although the study is associated with limitation of a small sample size, results obtained in this preliminary study are very promising to carry out further investigation in large number of patients. Another major limitation associated with the study is that we have not taken any gold standard like Quantiferon Gold test (QFT) to define LTBM cases; this is because of the low sample volume available for test $(1-1.5 \mathrm{~mL})$, whereas a CSF volume of more than $3 \mathrm{~mL}$ is required to perform QFT test. However, further studies can be planned to investigate the QFT response in Rv2623 positive cases.

\section{Conclusion}

The results of the present study suggest that Rv2623 may be useful as a diagnostic biomarker for latent and active TBM infections. Further investigation in a large number of samples along with its comparison with gold standards like QFT-G assay is needed to establish its role as a diagnostic biomarker of latent and active TBM.

\section{Acknowledgments}

The authors have no conflict of interests to declare. The work was funded by Central India Institute of Medical Sciences, Nagpur, India.

\section{References}

[1] World Health Organization, Global Tuberculosis Control: WHO Report, 2011, http://www.who.int/tb/publications/global_report/ 2011/en/.

[2] J. M. K. Murthy, "Tuberculous meningitis: the challenges," Neurology India, vol. 58, no. 5, pp. 716-722, 2010.

[3] R. S. Kashyap, K. M. Dobos, J. T. Belisle et al., "Demonstration of components of antigen 85 complex in cerebrospinal fluid of tuberculous meningitis patients," Clinical and Diagnostic Laboratory Immunology, vol. 12, no. 6, pp. 752-758, 2005.

[4] A. V. Mudaliar, R. S. Kashyap, H. J. Purohit, G. M. Taori, and H. F. Daginawala, "Detection of $65 \mathrm{kD}$ heat shock protein in cerebrospinal fluid of tuberculous meningitis patients," $B M C$ Neurology, vol. 6, article 34, 2006.

[5] G. Thwaites, T. T. H. Chau, N. T. H. Mai, F. Drobniewski, K. McAdam, and J. Farrar, "Tuberculous meningitis," Journal of Neurology Neurosurgery and Psychiatry, vol. 68, no. 3, pp. 289299, 2000.

[6] A. R. Rich and H. A. McCordick, "The pathogenesis of tuberculous meningitis," Bulletin of John Hopkins Hospital, vol. 52, pp. 5-37, 1933.

[7] G. E. Marx and E. D. Chan, “Tuberculous meningitis: diagnosis and treatment overview," Tuberculosis Research and Treatment, vol. 2011, Article ID 798764, 9 pages, 2011.

[8] R. H. Pando, "Modelling of cerebral tuberculosis: hope for continuous research in solving the enigma of the bottom Billion's disease," Malaysian Journal of Medical Sciences, vol. 18, no. 1, pp. 12-15, 2011.

[9] D. M. Monack, A. Mueller, and S. Falkow, "Persistent bacterial infections: the interface of the pathogen and the host immune 
system," Nature Reviews Microbiology, vol. 2, no. 9, pp. 747-765, 2004.

[10] I. Al-Orainey, "Diagnosis of latent tuberculosis: can we do better?" Annals of Thoracic Medicine, vol. 4, no. 1, pp. 5-9, 2009.

[11] J. E. Drumm, K. Mi, P. Bilder et al., "Mycobacterium tuberculosis universal stress protein Rv2623 regulates bacillary growth by ATP-binding: requirement for establishing chronic persistent infection," PLoS Pathogens, vol. 5, no. 5, Article ID e1000460, 2009.

[12] S. Ahmad, "Pathogenesis, immunology, and diagnosis of latent mycobacterium tuberculosis infection," Clinical and Developmental Immunology, vol. 2011, Article ID 814943, 17 pages, 2011.

[13] R. S. Wallis, M. Pai, D. Menzies et al., "Biomarkers and diagnostics for tuberculosis: progress, needs, and translation into practice," The Lancet, vol. 375, no. 9729, pp. 1920-1937, 2010.

[14] M. Y. Lin, Discovery of Dormancy Associated Antigens of Mycobacterium Tuberculosis, Leiden University Medical Center, Leiden, The Netherlands, 2009.

[15] R. O’Toole and H. D. Williams, "Universal stress proteins and Mycobacterium tuberculosis," Research in Microbiology, vol. 154, no. 6, pp. 387-392, 2003.

[16] S. M. Hingley-Wilson, K. E. A. Lougheed, K. Ferguson, S. Leiva, and H. D. Williams, "Individual Mycobacterium tuberculosis universal stress protein homologues are dispensable in vitro," Tuberculosis, vol. 90, no. 4, pp. 236-244, 2010.

[17] J. L. Flynn and J. Chan, "Tuberculosis: latency and reactivation," Infection and Immunity, vol. 69, no. 7, pp. 4195-4201, 2001.

[18] S. D. Schuck, H. Mueller, F. Kunitz et al., "Identification of T-cell antigens specific for latent mycobacterium tuberculosis infection," PLoS One, vol. 4, no. 5, article e5590, 2009.

[19] I. M. Monahan, J. Betts, D. K. Banerjee, and P. D. Butcher, "Differential expression of mycobacterial proteins following phagocytosis by macrophages," Microbiology, vol. 147, part 2, pp. 459-471, 2001.

[20] M. A. Florczyk, L. A. McCue, R. F. Stack, C. R. Hauer, and K. A. McDonough, "Identification and characterization of mycobacterial proteins differentially expressed under standing and shaking culture conditions, including Rv2623 from a novel class of putative ATP-binding proteins," Infection and Immunity, vol. 69 , no. 9, pp. 5777-5785, 2001. 


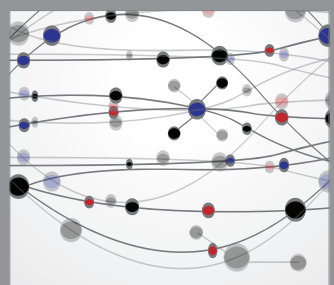

The Scientific World Journal
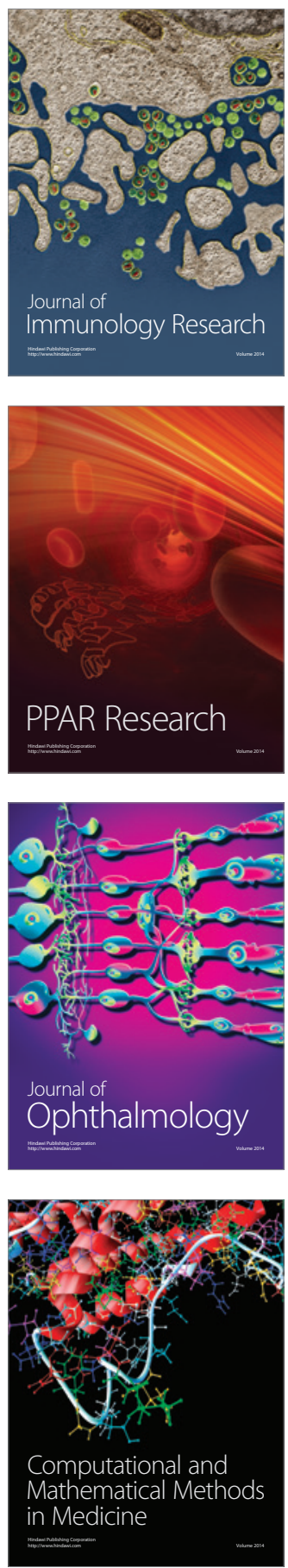

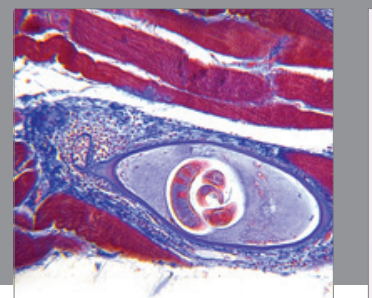

Gastroenterology

Research and Practice
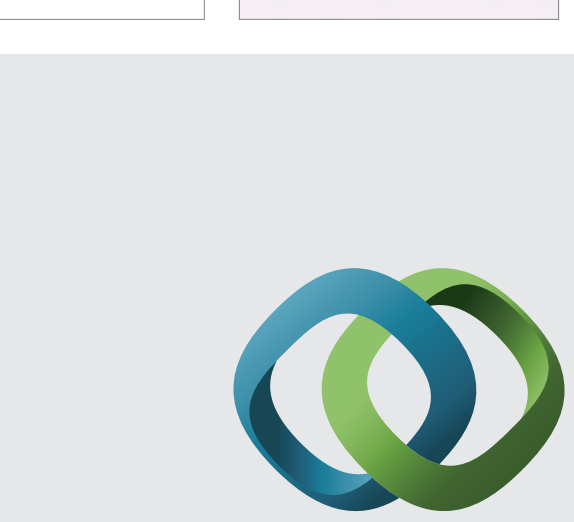

\section{Hindawi}

Submit your manuscripts at

http://www.hindawi.com
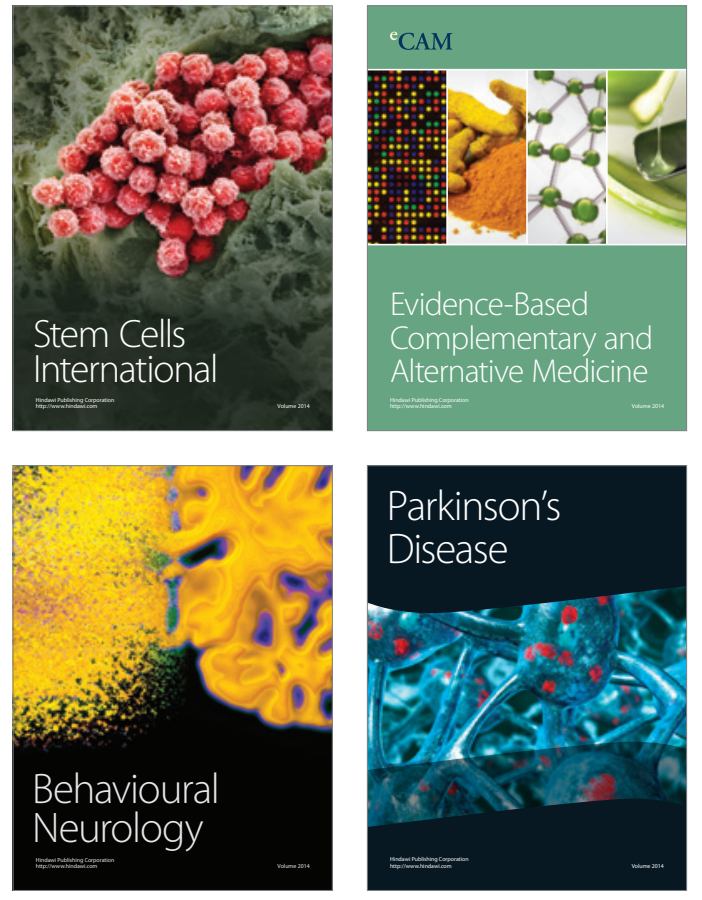
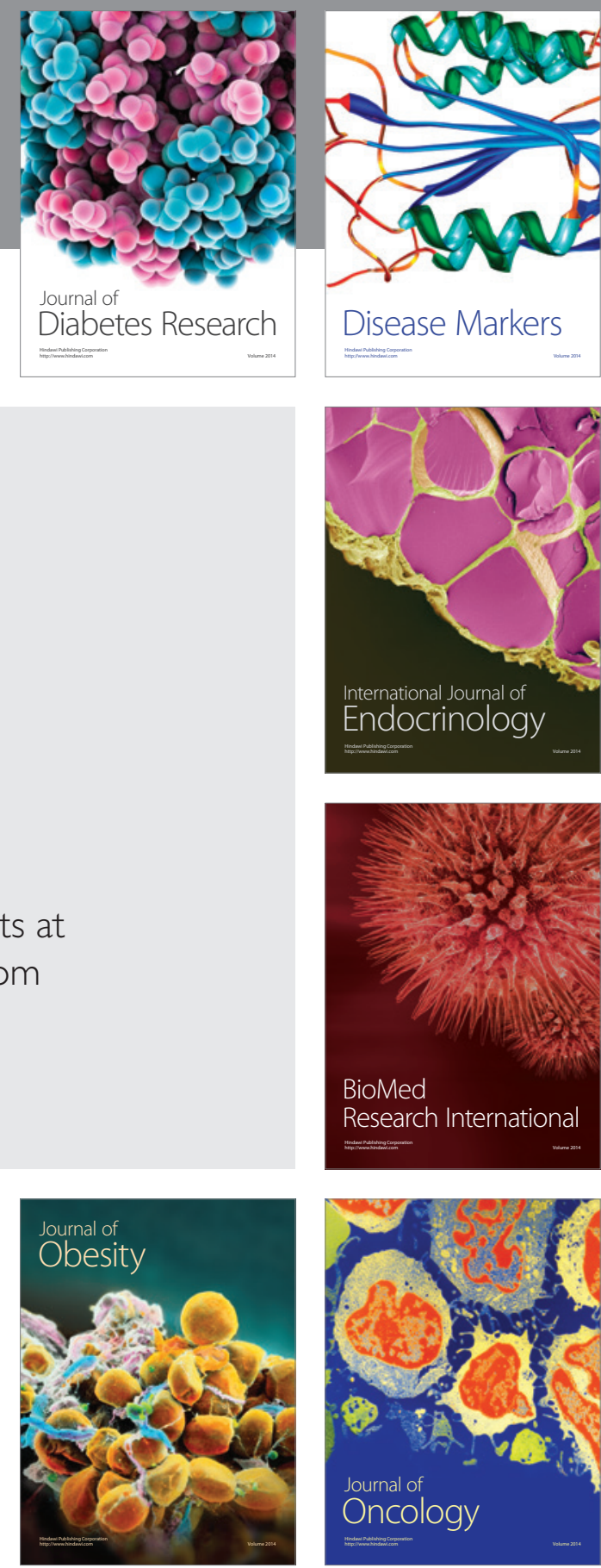

Disease Markers
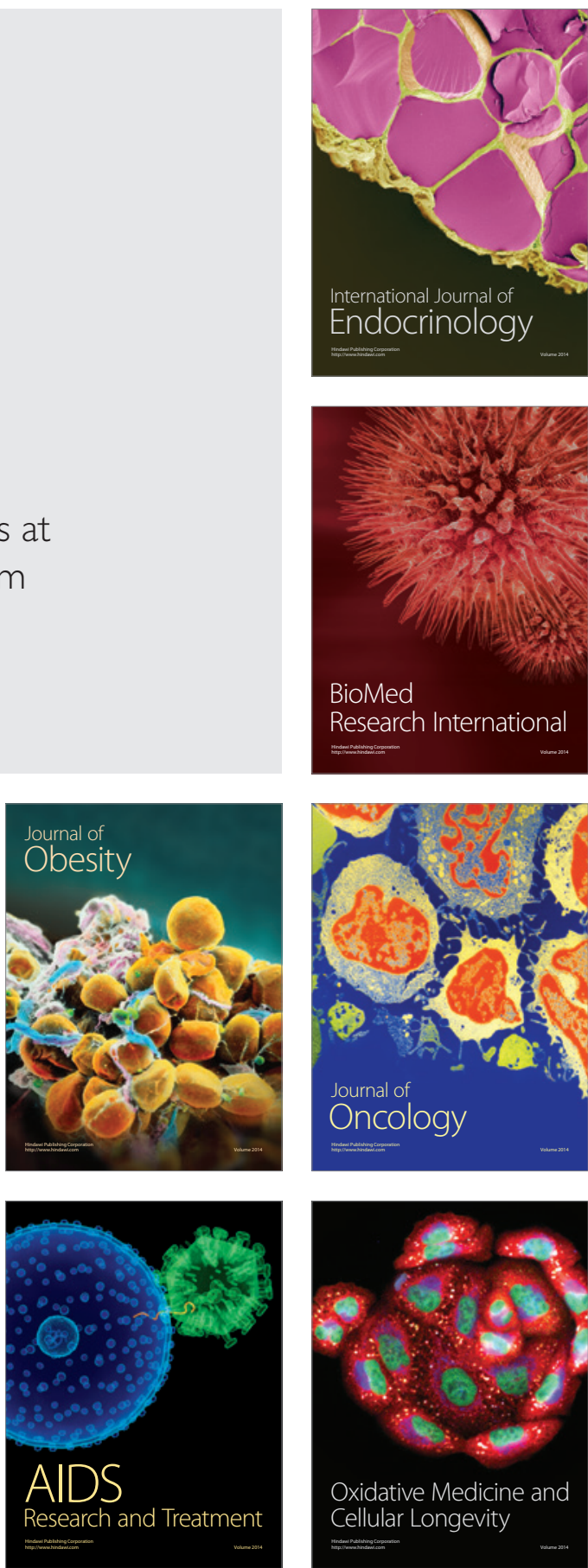